\title{
A Preamble-Based Broadcasting Technique for Wireless Ad Hoc Sensor Networks
}

\author{
Arun Kumar, Kai-Juan Wong \\ Emerging Research Lab, School of Computer Engineering, Nanyang Technological University, Singapore \\ E-mail: \{arun0020, askjwong\}@ntu.edu.sg \\ Received November 15, 2010; revised February 8, 2011; accepted March 16, 2011
}

\begin{abstract}
Broadcasting is a fundamental operation in any wireless networks, more so in wireless ad hoc sensor networks, where each sensor node has limited transmission range and battery power. Although broad-casting in wireless ad hoc sensor networks has many advantages but it can cause serious problems like-broadcast storm, which could cause a lot of contention, redundant, retransmission, collision and most importantly, drain immense amount of energy from limited battery powered sensor nodes. In this work, our objective is to reduce the number of retransmission and energy consumption of sensor nodes by using the duty cycle property of wireless ad hoc sensor networks. We propose a preamble-based broadcasting technique for wireless ad hoc sensor networks. We show that in dense wireless ad hoc sensor networks a small size preamble can give maximum network-wide data dissemination rather than using the large preamble, which will only consume immense amount of energy during packet reception.
\end{abstract}

Keywords: Broadcast, Flooding, Wireless Ad Hoc Sensor Network

\section{Introduction}

Wireless ad hoc sensor networks are a set of wireless sensor nodes (mobile or static) which communicate with each other without any pre-existing routing infrastructure for communication, and mostly rely on their neighbor nodes to relay their data up to the destination node instead of directly communicating with the destination node or sink. These sensor nodes have several resource constraints such as limited memory, battery power, and signal processing, computational and communication capabilities. These tiny nodes are mainly deployed in remote areas and work unattended for many years, making battery life of the sensor node a major area of concern.

A large-scale wireless ad hoc sensor network can consist of thousands of sensor nodes and are helpful in many situations [1], such as disaster relief missions, battlefield communication facilities, infrastructure protection and scientific exploration [1-3]. Wireless data transmission consumes more energy than data processing in a sensor node and the situation become worse in dense wireless networks where broadcasting of data is needed. Even a single bit transmission consumes the same energy as that needed for processing thousand operations in a typical sensor node.

Wireless ad hoc sensor networks have broadcast as one of its most fundamental service. Broadcast provides maximum message propagation across the whole network and serves high-level operations, making it critical to the overall network design. Although, broadcasting in wireless ad hoc sensor networks have many advantages, it can cause serious problems like broadcast storm problems [4], which could cause a lot of contention, redundant retransmission, collision and most importantly waste immense amount of energy. A possible solution is that the sensor nodes alternate between active and dormant states, which help them to conserve energy and extend the network lifetime. Simple broadcasting in this environment, where a node goes to active and dormant states periodically can be very challenging if there are not enough nodes in the active state and when the source wants to send data.

In this paper, we propose a preamble based broadcasting technique for wireless ad hoc sensor networks, while considering the challenges raised by the broadcast storm problems in such networks. The pro- posed technique is implemented and studied thoroughly, taking mobile node in the wireless ad hoc sensor networks. A probability-based technique is also implemented and studied to 
compare with our technique. The set of performances matrics chosen for fair comparison are reachability, throughput, average number of duplicate packets and energy consumption.

The rest of the paper is configured as follows. Section 2 gives a background of broadcasting techniques for wireless networks. In Section 3, we present our proposed preamble-based broadcasting technique. The simulation platform used, the parameters used during the simulation and the matrics used for comparison are given in Section 4. Section 5 gives a thorough discussion of the results. Section 6 concludes the paper and gives suggestions for future work.

\section{Background}

There are many proposed approaches for broadcasting in which, flooding is one of the earliest broadcasting mechanisms in wired and wireless networks. In flooding, each node rebroadcasts the packets when received for the first time, already received packets just dropped. Though flooding is simple, it consumes most of the network resources as a large number of duplicate packets flooded repeatedly. This can leads to serious redundancy, contention and collision in any wireless network and is referred as the "broadcast storm problem" [4].

The authors in [4] have classified broadcasting schemes into probabilistic, counter-based, distance based, location-based and cluster-based. In the probabilistic scheme, a host will rebroadcast the message with the maximum probability $p=1$, if it receives for first time; otherwise every time the probability would be decreased. In the counter-based scheme, a host will drop the packet if the number of duplicates received crosses a threshold. In the distance-based scheme, a host will rebroadcast the packet only if the sender and receiver distance is larger than a threshold. The location-based scheme rebroadcast the message if the additional coverage due to this broadcast is bigger than a threshold. Finally, in the clusterbased scheme, clusters are created using efficient cluster selection algorithms and the cluster head and gateways do the rebroadcast. An alternate classification of broadcasting techniques found in [5] has four categories: simple flooding, probability-based, area-based, and neighbor knowledge-based schemes.

Several duty cycle aware broadcast protocols have been proposed [6-9] in recent years for wireless sensor networks. Duty cycle aware broadcast [6] shows that conventional broadcast protocol cannot cover the whole network in an acceptable timeframe and the performance degrades in low duty cycle environment. The protocol tried to balance between latency and efficiency with coverage guarantees by remodeling the broadcast problem for a low duty cycle environment.

In Opportunistic flooding [7] the decision to forward a packet is based on probabilistic forwarding decisions at the sender side, considering the delay distribution of next-hop nodes. A new node joining the network shares its schedule with the entire neighborhood and the process of sharing the schedule is referred to as low duty cycle rendezvous. To reduce redundancy in transmission and flooding delay, only opportunistically early packets are forwarded using links outside the energy optimal tree. A forwarder selection method to overcome the hidden terminal problem and a link-quality based back off method to resolve simultaneous forwarding operations are proposed by the author. Opportunistic flooding considers the working schedules of sensor nodes as fixed. The algorithm assumes that the network is locally synchronized using MAC layer time stamping technique, which makes the algorithm complex, as synchronization itself is a complex problem in big area network.

Asynchronous Duty-cycle Broadcasting (ADB) [8] is integrated with the MAC layer and uses the information available at this layer, unlike traditional multi-hop broadcast which operates above the MAC layer. ADB optimizes the broadcast progress in the network at the level_of transmission from the node to each neighbor individually. In ADB, a sender first transmits unicast packet to a node when it is in active state and through the acknowledgement, it learns if the neighbor has been reached through the broadcast. It is integrated with the RI-MAC [8] to announce its wake up with the beacon packets. ADB can efficiently perform in unicast but the performance in broadcast traffic scenarios is not thoroughly studied.

The solution to this problem can be made simpler by making use of a tree to flood packets. But this solution can be very fragile $[9,10]$ because a single node failure (parent node) can prevent all its sub trees nodes from receiving messages, even though the network is fully connected. These tree solutions can be energy efficient at the cost of long delays, as the packets are always forwarded via a single route.

Unfortunately, more or less all the above mentioned protocols are concerned mostly about the quality of service in wireless ad hoc sensor network and do not consider the challenges raised by broadcasting such as redundant transmission, collision and contention.

In this paper, we have considered asynchronous duty cycling nodes during the simulation of our proposed broadcasting scheme. We have used a small preamble of variable length to overcome the vast energy consumption of sensor nodes and significantly reduce the number of 
retransmissions in wireless ad hoc sensor networks.

\section{Preamble-Based Broadcasting}

Wireless ad hoc sensor networks have an important property that many sensor nodes alternate between active and dormant states, which help them to conserve energy and extend the network lifetime. In a dense area networks there is a greater chance of a node being in an active state to listen to the channel at a particular time, in contrast to a sparse area network. Simple flooding in dense area network, where more nodes are active at a time near the relay node will only cause energy consumption rather than covering additional areas in the network, resulting in unnecessary retransmission of pack- ets and collisions.

We have used small preambles in dense wireless ad hoc sensor networks before the data packet. This small preamble before the data packet in the dense area network will be listened by a subset of nodes present in the radio range of a relay node or sender node. The subset of sensor nodes that received the data packet then rebroadcasts the packet using the same preamble length. This practice of sending a small preamble before the data packet reduces the number of rebroadcasts as well as the overall energy consumption in the network, as only a subset of nodes will listen to the preamble and receive the data packet. Each sensor node rebroadcast the packet only after a random delay to make the timing of rebroadcasting differentiated.

In a highly dense area network, a very small preamble can give maximum data dissemination in contrast to a comparatively large preamble required in a sparse area network where there are lesser chances that a node is active in the neighborhood of the relaying sensor node or the sender node. This will save most of the rebroadcasts and conserve maximum energy in dense area networks when compared to sparse networks, where small preamble cannot guarantee minimum number of node for network wide data dissemination.

Figure 1 compares simple and preamble based flooding. Figure 1(a) shows the effect of simple flooding in a wireless ad hoc network where every sensor node is involved in flooding and number of retransmission is large. Arrows are pointed both sides to show that a node is forwarding a packet to its neighbor node as well as getting the packet from its neighbor node to whom it forwarded the packet before. Figure 1(b) shows broadcasting using preamble, based technique in duty cycle networks, where a subset of sensor nodes receives a packet every time a node broadcasts it, thus providing network wide coverage with minimum retransmission of packets.

The relevance and benefits of using a small preamble

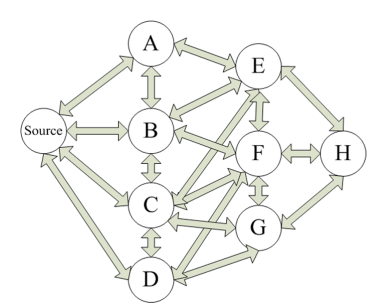

(a)

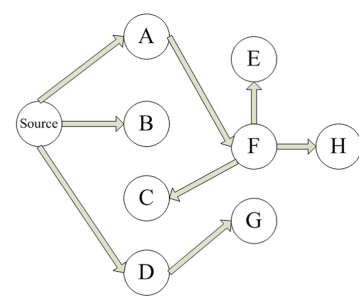

(b)
Figure 1. Different flooding structures. (a) Original flooding (b) Flooding using preamble technique in duty cycle network.

before broadcast data packets to get network wide dissemination can be justified in following steps:

- Sensor nodes in the wireless ad hoc sensor net- works are tiny devices with several constraints, such as limited radio range (20 - $30 \mathrm{~m}$ typically), memory, battery power, computation and commu- nication capabilities. These devices are mainly de- ployed in remote areas and work non-stop $24 \times 7$ unattended for many years. As a result the main focus is on saving the battery power of the sensor node.

- Broadcasting method such as simple flooding can cause severe energy consumption on already bat- tery power limited nodes.

- Redundant rebroadcast of message is undesirable in both dense and sparse wireless ad-hoc sensor networks. Redundant rebroadcast cause immense amount of energy wastage and at the same time is not of much help in distributing the message throughout the network.

- Broadcast packets in wireless ad hoc sensor networks are generally used for route discovery or for periodically updating the routing table. In this case, when broadcast- ing is needed periodically, simple flooding can consume most of the network resources, even before the actual data packet reaches to its destination (sink).

\section{Simulation and Analysis}

We have implemented our protocols using the QualNet 5.0 simulator. QualNet can be used to optimize new and existing models, analyze the performance of networks, perform cross-layer optimization of wired or wireless network stacks or design a network in QualNet Developer to perform capacity, performance, and scalability analyses. We have modified several layers of QualNet to implement BMAC and our proposed protocol.

\subsection{Application Layer Implementation}

The Application Layer of QualNet 5.0 does not provide 
broadcast based traffic-generating protocol, it provides several unicast and multicast based traffic generating protocol such as CBR, VBR, MCBR, CELLU-LARABSTRACT-APP and GSM.

We have implemented our own broadcast traffic generating protocol on QualNet 5.0. This enables the nodes present in the network to broadcast data packet network-wide. A node broadcasts the data packet just after receiving it from any other node, without any delay. Every broadcast packet is 32 byte in size and contains a sequence number as well as a timestamp, which contains the time the packet was sent by the other node.

\subsection{MAC Layer Implementation}

QualNet 5.0 MAC library provides several MAC Layer protocols, for both wireless and wired category and provides the flexibility of running different MAC protocols (wired and wireless mix) on a node, if a node has multiple interfaces. Some examples of the MAC Layer protocols available in qualnet are as ALOHA, CSMA, TDMA, GENERICMAC, MAC802.3, MAC802.15.4 and MACDOT11.

Although, QualNet 5.0 has a variety of MAC Layer protocols in its library, it lacks any Energy Aware MAC Layer protocol such as S-MAC, D-MAC, BMAC, $\mathrm{X}-\mathrm{MAC}, \mathrm{RI}-\mathrm{MAC}$ and others. We have tried to fill this gap by implementing the most popular energy aware MAC layer protocol, BMAC in QualNet 5.0. To implement BMAC, the physical layer of QualNet is modi- fied to simulate sleep and wakeup states of radio.

\subsection{Physical Layer Implementation}

The Physical Layer of QualNet 5.0 does not model the sleep and waking up states of the radios, which is assumed to be either in the transmission or receiving modes. The physical layer model is modified to allow the radios to go on sleep mode, when nothing is on the channel after a predefined time or after receiving the data packet. The channel is sensed periodically and if anything is detected on channel, the node will wake up the radio. Switching from sleep to idle takes significant time, which is considered as the waking up time and the state called as waking up state. Adding the sleep state in the physical layer will help the nodes in energy conservation by allowing them to control when to switch ON and OFF the radio. The physical layer of QualNet is modeled to be in one of the Sleep/Waking-up/Idle/Transmit/Receive states. Successful implementation of these states is mportant for the correct implementation of a duty cycling MAC protocol in QualNet Simulator.

\subsection{Broadcasting in Qualnet}

The simulation is a collection of network nodes, each with its own protocol stack parameters and statistics accessible from the "Node" structure. There are two types of events in QualNet: packet events and timer events.

The two types of messages/events that represent a broadcast event are discussed below. These are data structures that hold information on broadcast event type and associated data.

1) Packet events (data or control)_-simulate exchange of data packets between layers or between nodes.

Timer events - simulates time-outs, allows broadcast protocol to schedule events in a future time; internal to a protocol. Timer events are set and received within a protocol and they do not travel through the protocol stack.

QualNet uses a layered architecture, similar to that of the TCP/IP network protocol stack. Within that architectture, data moves between adjacent layers. QualNet protocol stack consists of, from top to bottom, the Application, Transport, Network, Link (MAC) and Physical Layers. Adjacent layers in the protocol stack communicate via well-defined APIs shown in Table 1, and generally, layer communication occurs only between adjacent layers, as shown in Figure 2.

\subsection{Simulation Parameters}

\subsubsection{Parameters for Preamble-Based Broadcasting}

As stated earlier, we have simulated our work on QualNet 5.0. Due to the several constraints on wireless sensor nodes, we have fixed some parameters used in simulation, such as the radio range $(20.023 \mathrm{~m})$, data rate (250 kbps) and mobility of nodes as $2 \mathrm{~m} / \mathrm{s}$. Certain parameters are determined based on the CC2420 transceiver [12], such as transmit power consumption of radio $(57.4 \mathrm{~mW})$, receiver power consumption $(62.1 \mathrm{~mW})$, and power consumption during sleep mode $(1.41 \mathrm{~mW})$.

For the simulations, node 1 is always chosen as source node, generating the broadcast packet (1 packet/sec) and all nodes are mobile including the source node. Preamble

Table 1. Message-related API functions.

\begin{tabular}{|c|c|}
\hline MESSAGE_Alloc( ) & $\begin{array}{l}\text { Allocate a message and provide it } \\
\text { with standard event, layer, protocol } \\
\text { info }\end{array}$ \\
\hline MESSAGE_InfoAlloc( ) & $\begin{array}{l}\text { Allocate additional user-specified } \\
\text { space for optional information about } \\
\text { the event }\end{array}$ \\
\hline MESSAGE_PacketAlloc( ) & $\begin{array}{l}\text { Allocate space for the packet within } \\
\text { the message }\end{array}$ \\
\hline MESSAGE_Send( ) & $\begin{array}{l}\text { Send the message as an event to the } \\
\text { specified layer and protocol }\end{array}$ \\
\hline MESSAGE_Free() & $\begin{array}{l}\text { Free the message, once it has } \\
\text { reached its final destination }\end{array}$ \\
\hline
\end{tabular}




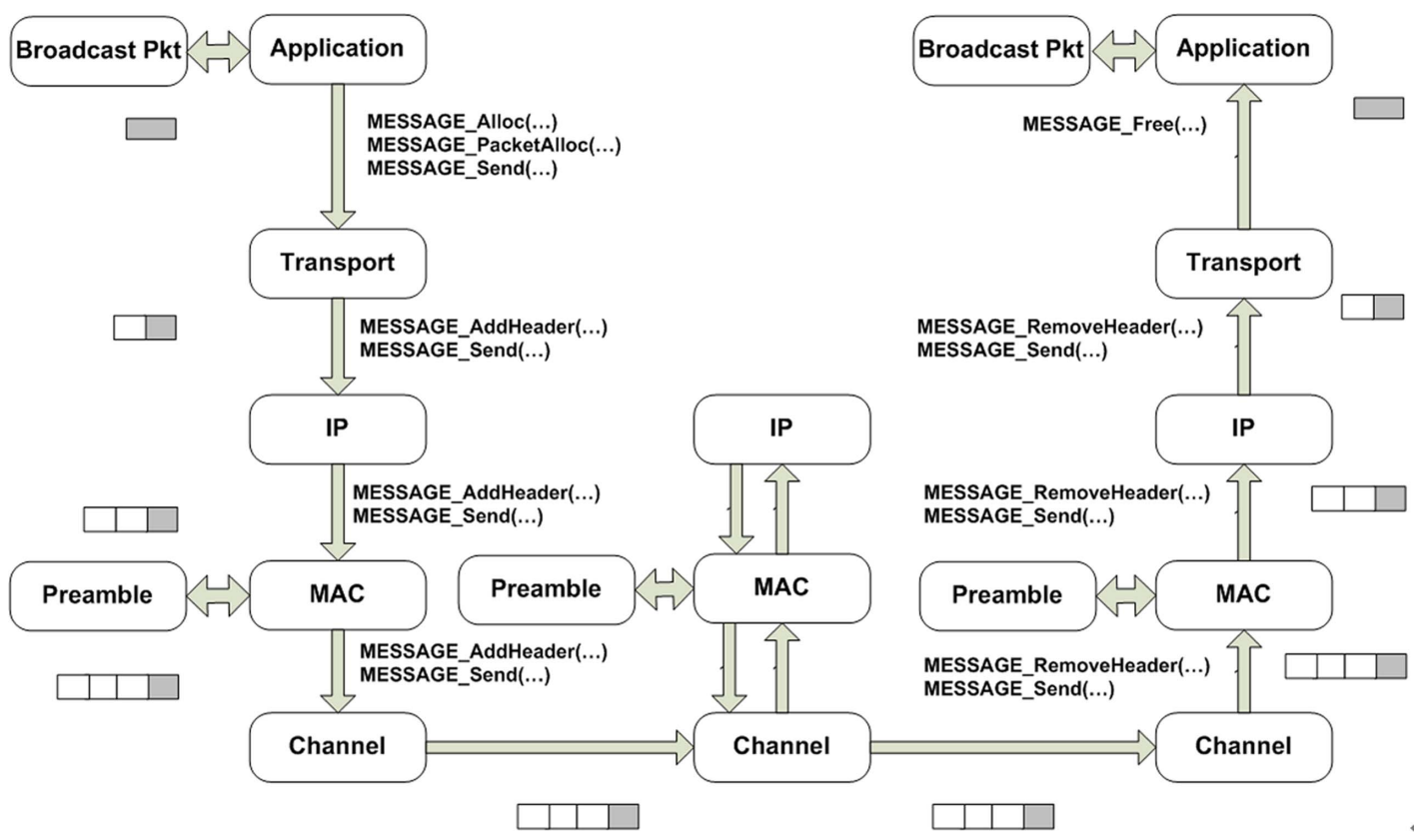

Figure 2. Broadcast packet protocol stack in qualnet.

length ranges from $4 \mathrm{~ms}$ to $21 \mathrm{~ms}$. Some important parameters used during simulation are given in the Table 2 .

\subsubsection{Parameters for Probability-Based Broadcasting} The technique, probability based technique for broadcasting is also simulated using QualNet 5.0. Several parameter used during simulation are same as preamble based techniques such as the radio range $(20.023 \mathrm{~m})$, data rate (250 kbps), mobility of nodes $(2 \mathrm{~m} / \mathrm{s})$ and the power consumption. Nodes in the network are not duty cycled and CSMA is used as MAC layer protocol.

Broadcast packets are sent using different probabilities, ranging from 0.2 to 1 . Node 1 is always chosen as source node generating the broadcast packet (1 packet/sec) and all nodes are mobile including the source node. Some important parameters used during simulation are given in the Table 3 .

A random delay of a few microseconds is taken on each node before rebroadcasting the packet, to make the timing of rebroadcasting differentiated in both the broadcasting technique.

\subsection{Performance Metrics}

The set of performance metrics used for analysis are as follows:

- REachability (RE): We define the reachability as the number of sensor nodes receiving the broadcast message divided by the total number sensor nodes that are reachable, directly or indirectly, from the source node.

- Throughput: We define the throughput as the average number of unique packet successfully received by a node over the total number of unique packets send by the source node.

- Average Duplicate Packet: We define the average duplicate packet as the average number of duplicate packet received by a node during the simulation time.

- Energy Consumption: Energy consumption for a particular network density is calculated as the average energy consumption of a node during the simulation time.

Table 2. Parameters used in simulation.

\begin{tabular}{ll}
\hline Application Layer & Broadcast \\
MAC Layer & BMAC \\
Path Loss Model & Two-Ray \\
Bandwidth & $250 \mathrm{kbps}$ \\
Payload Size & 32 bytes \\
Terrain & $50 \times 50,100 \times 100,200 \times 200$, \\
Node Placement & $300 \times 300,400 \times 400,500 \times 500$ \\
Radio Range & Uniform \\
Node Numbers & $20.023 \mathrm{~m}$ \\
Mobility & 250 \\
Wakeup Interval & $2 \mathrm{~m} / \mathrm{sec}$. \\
Simulation Time & $20 \mathrm{~ms}$ \\
Iterations & $500 \mathrm{sec}$. \\
\hline
\end{tabular}


Table 3. Parameters used in simulation.

\begin{tabular}{ll}
\hline Application Layer & Broadcast \\
MAC Layer & CSMA \\
Path Loss Model & Two-Ray \\
Bandwidth & $250 \mathrm{kbps}$ \\
Payload Size & 32 bytes \\
Terrain & $50 \times 50,100 \times 100,200 \times 200$, \\
Node Placement & $300 \times 300,400 \times 400,500 \times 500$ \\
Radio Range & $20.023 \mathrm{~m}$ \\
Node Numbers & 250 \\
Mobility & $2 \mathrm{~m} / \mathrm{sec}$. \\
Simulation Time & $500 \mathrm{sec}$. \\
Iterations & 10 \\
\hline
\end{tabular}

\section{Results and Discussion}

Network Sizes shown in figures are $1 \times 1,2 \times 2,4 \times 4,6$ $\times 6,8 \times 8,10 \times 10$ units, where a unit represents 50 meter in length.

\subsection{Reachability}

Figure 3 and Figure 4 show the percentage nodes reached versus network size, comparing the preamble-based broadcasting with the probability-based broadcasting. We observe that in dense area network broadcasting a packet with a small preamble or with a less probability is sufficient to disseminate the broadcast packet throughout the network. In contrast, as the network becomes sparse, a broadcast packet needs to be sent with a longer preamble or with a high probability. We observe that there is no significant difference in our preamble based broadcast as compared with the probability-based broadcast in terms of reachability. Our scheme provides higher reachability in dense area network and is a better solution in term of reachability in dense area wireless ad hoc sensors networks.

\subsection{Throughput}

Figure 5 and Figure 6 show the percentage throughput versus network size, comparing preamble-based broadcasting with probability-based broadcasting. We observe that in a very dense area network probability-based broadcasting fails to achieve high throughput due to the high collisions of packets. It is observed that in dense area networks preamble-based scheme achieves high throughput even if not sent with a long preamble. But when the network becomes sparser probability-based scheme achieves high throughput because the nodes are at all times active in the network. The abrupt drop in

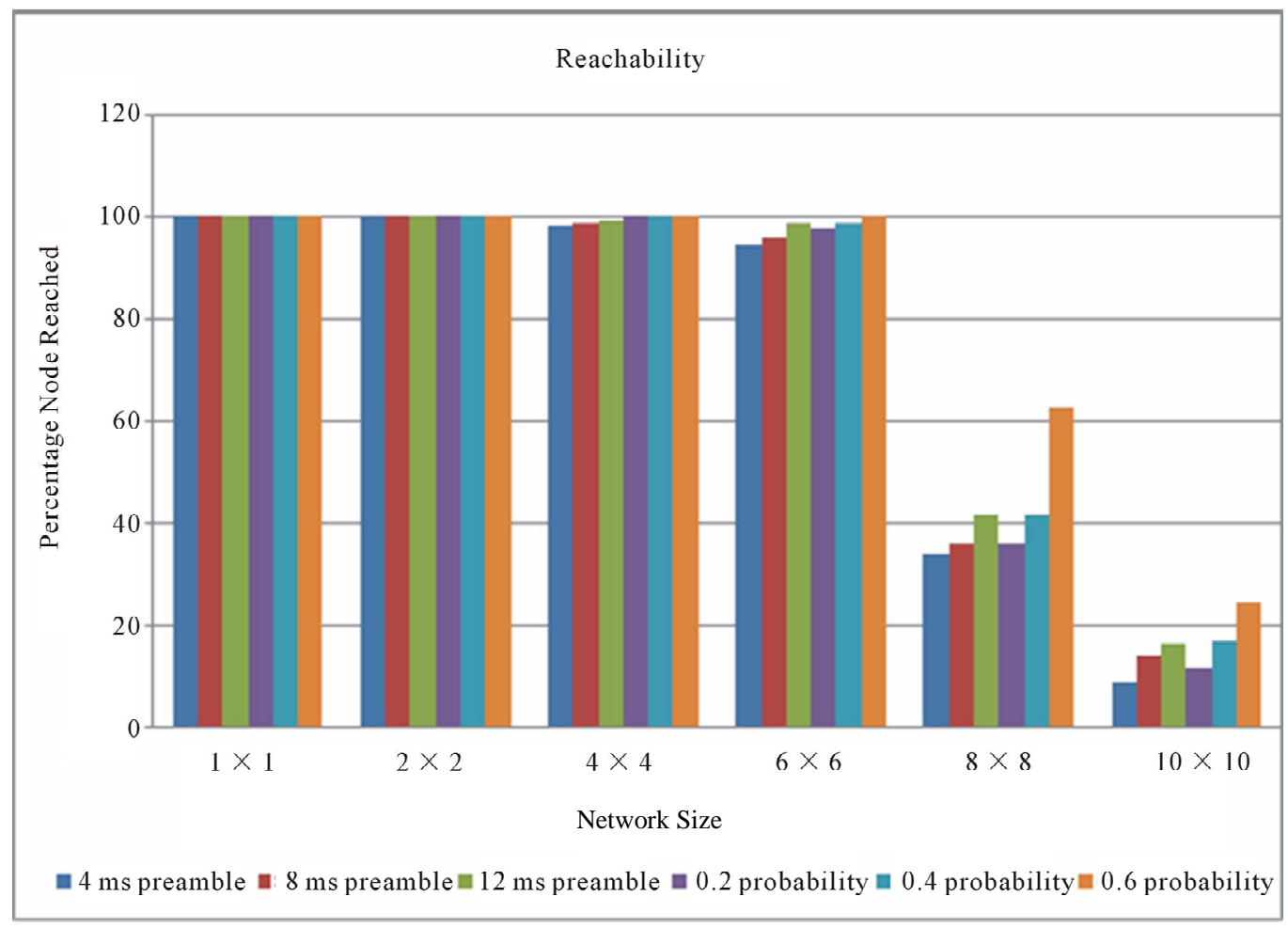

Figure 3. Percentage node reached vs network size. 


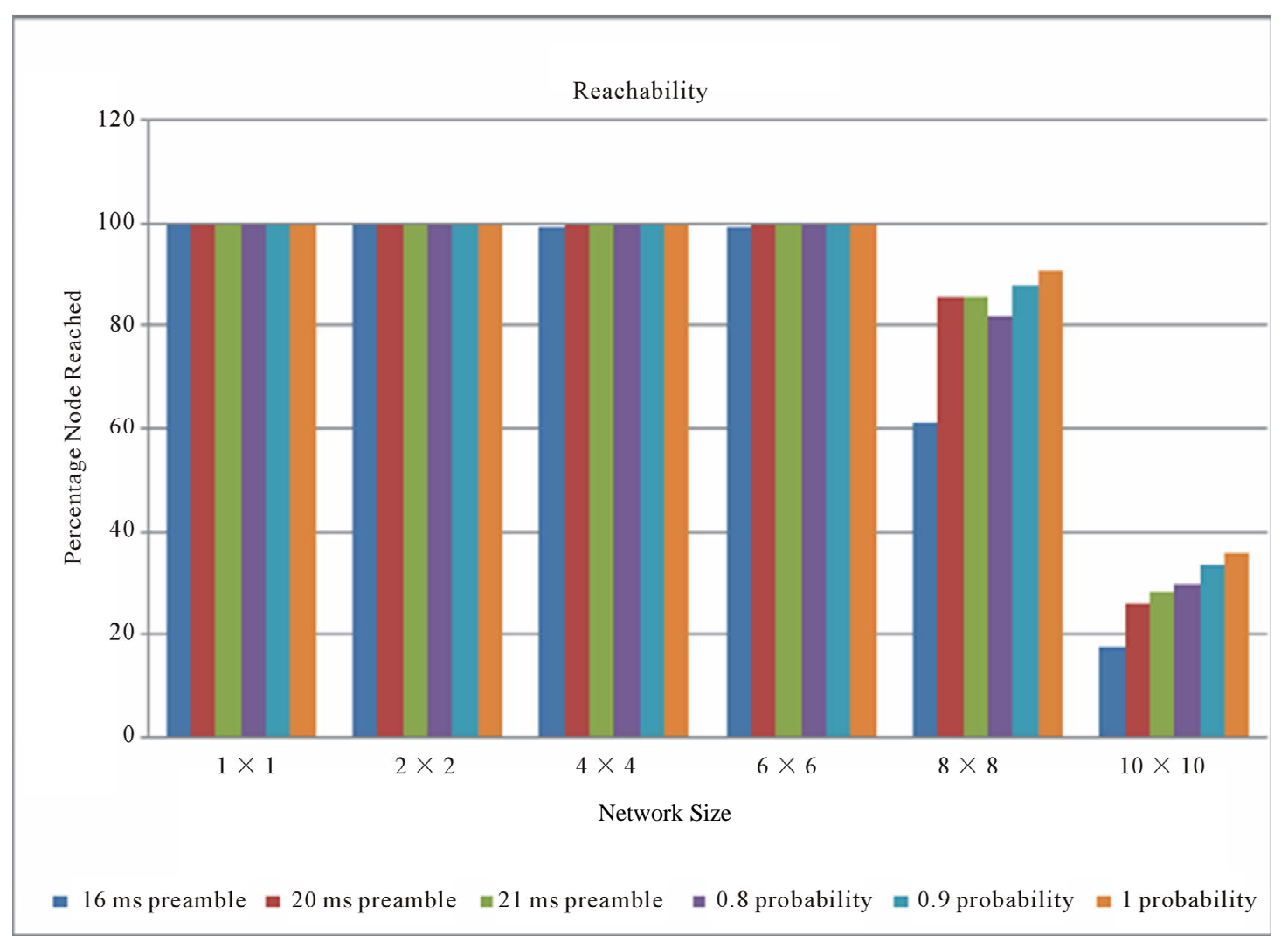

Figure 4. Percentage node reached vs network size.

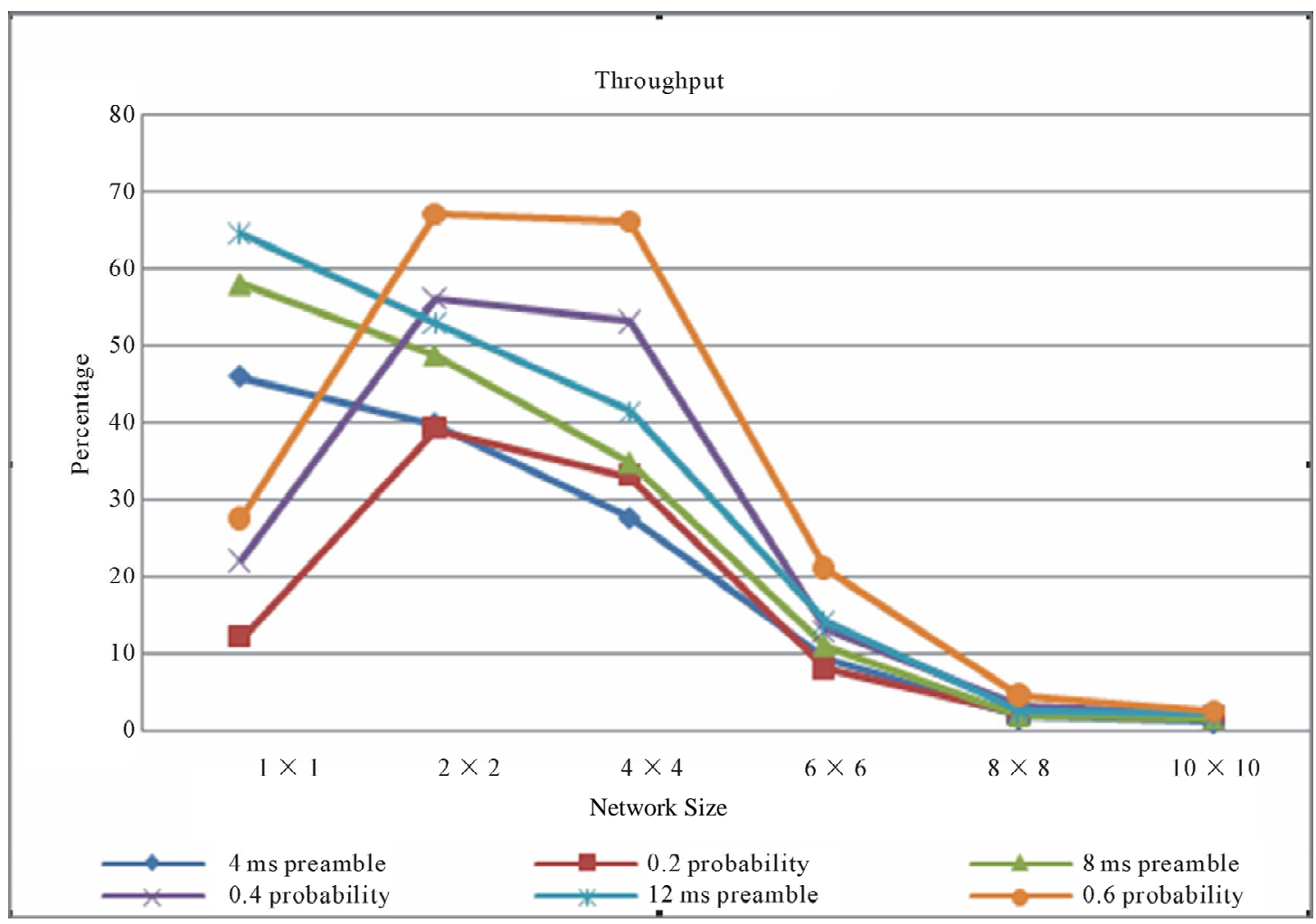

Figure 5. Percentage throughput vs network size. 


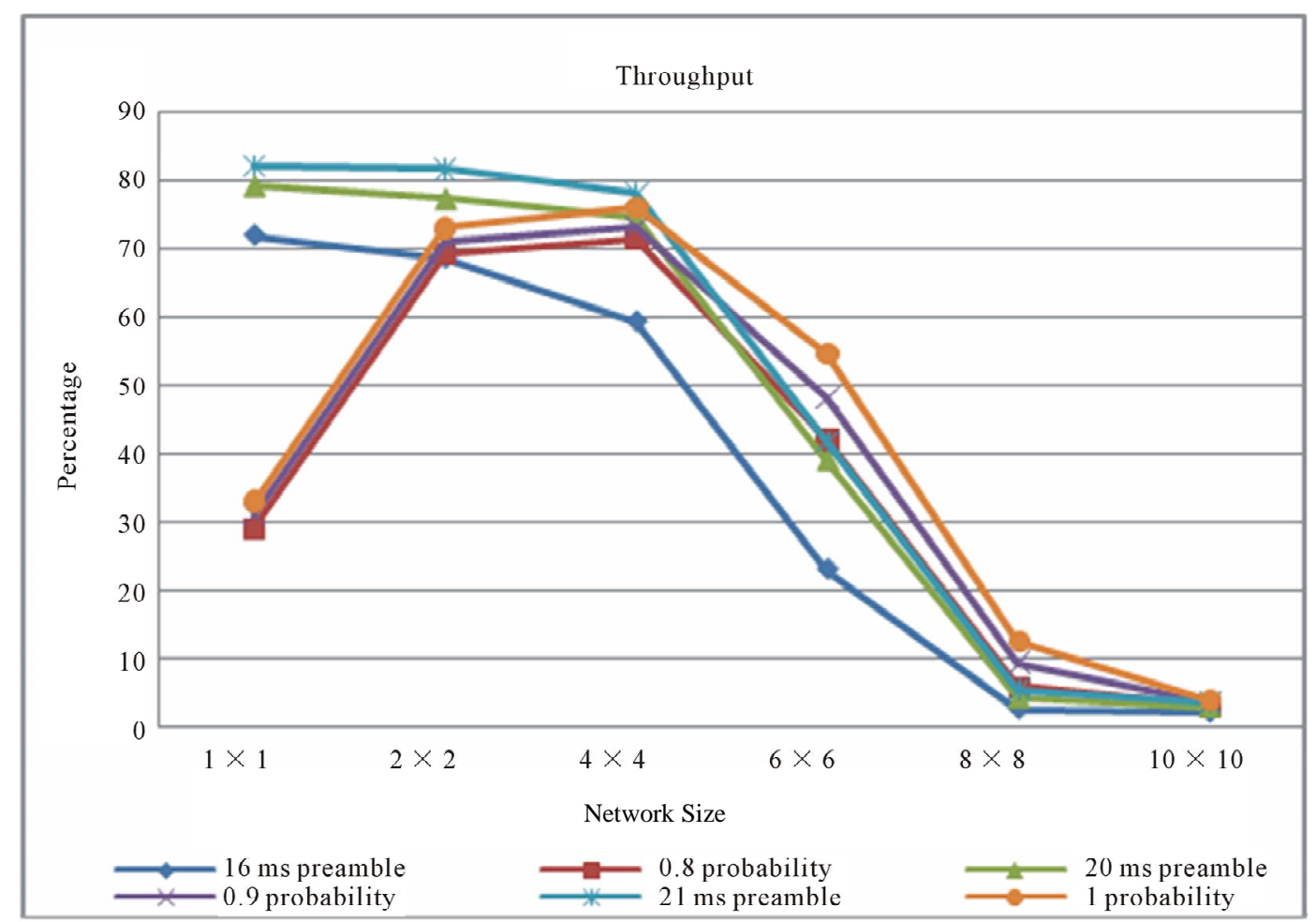

Figure 6. Percentage throughput vs network size.

throughput in sparse area networks is due to minimum reachability. The high throughput in the probabilitybased schemes comes at a cost of high-energy consumption and duplicates packets. It is observe that our preamble-based scheme provides a better throughput while consuming less energy.

\subsection{Average Duplicate Packet}

Figure 7 and Figure 8 show the duplicate packets/node versus network size, comparing the preamble-based broadcasting with the probability-based broadcasting. We observe that in a very dense area network $(1 \times 1)$ nodes received less duplicates packet when using probability-based broadcasting, due to the high number of collision and packet loss. We observe that in probabilitybased scheme, the duplicate packet reception is three to four times higher than the preamble-based scheme in various network sizes. As the network becomes sparse, nodes receives less duplicates packets in both the schemes, though in probabilistic scheme duplicate packet reception is higher.

\subsection{Energy Consumption}

Figures 9-11 show the energy consumption (mj/node) versus network size, comparing the preamble-based broadcasting with the probability-based broadcasting. We observe that energy consumption per node in probability-based scheme is always high in contrast to the preamble-based scheme. We conserve the energy per node in preamble-based broadcasting by allowing the node to go to dormant state when nothing is on the channel and save the energy at transmission node and receiver node by transmitting a small preamble and receiving a small preamble respectively before the data packet. It is observe that preamble-based scheme is best suited in term of energy conservation in the nodes as compared to the probability-based scheme. Energy consumptions per node are always high in probability-based scheme due to its always active node in the network and unnecessary energy consumption in nodes during the idle mode.

\section{Conclusions}

In this paper, we have proposed a preamble-based broadcasting technique for wireless ad hoc sensor networks. The proposed preamble-based broadcast technique is implemented and studied thoroughly, considering mobile nodes in wireless ad hoc sensor networks. An existing probability-based technique is also implemented and studied to compare with our preamble-based technique. We observe that our scheme provides high reach- 


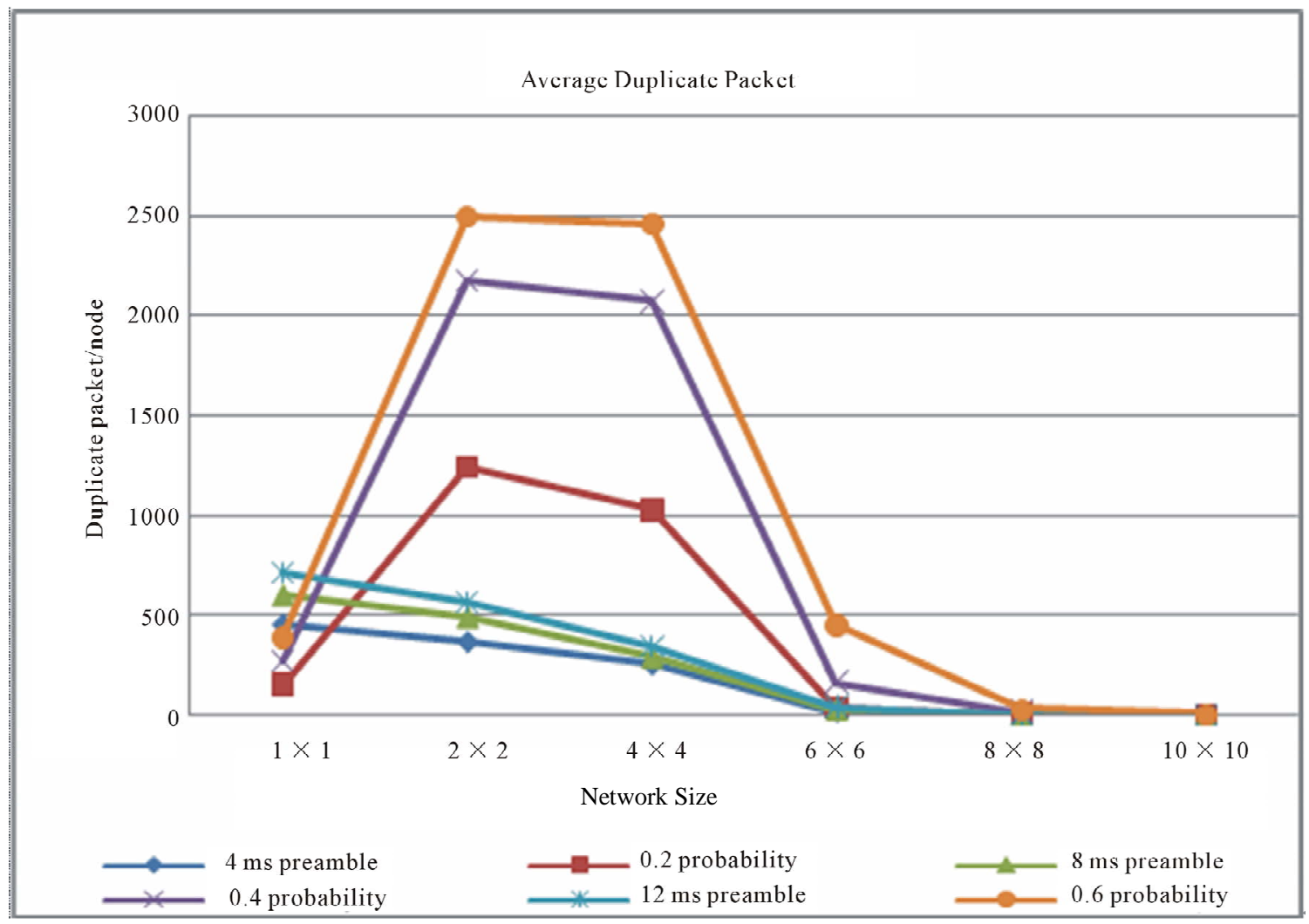

Figure 7. Duplicate packets/node vs network size.

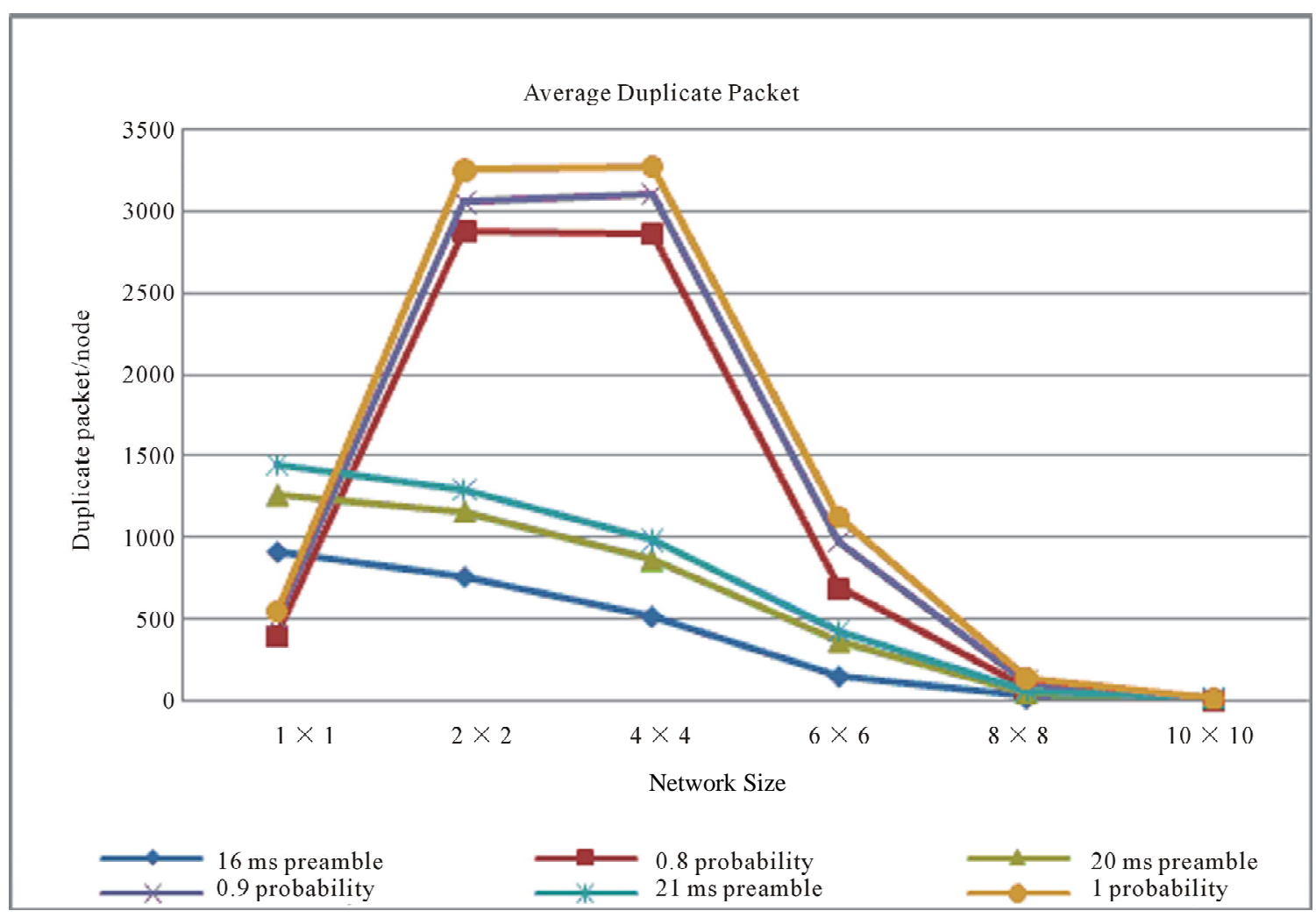

Figure 8. Duplicate packets/node vs network size. 


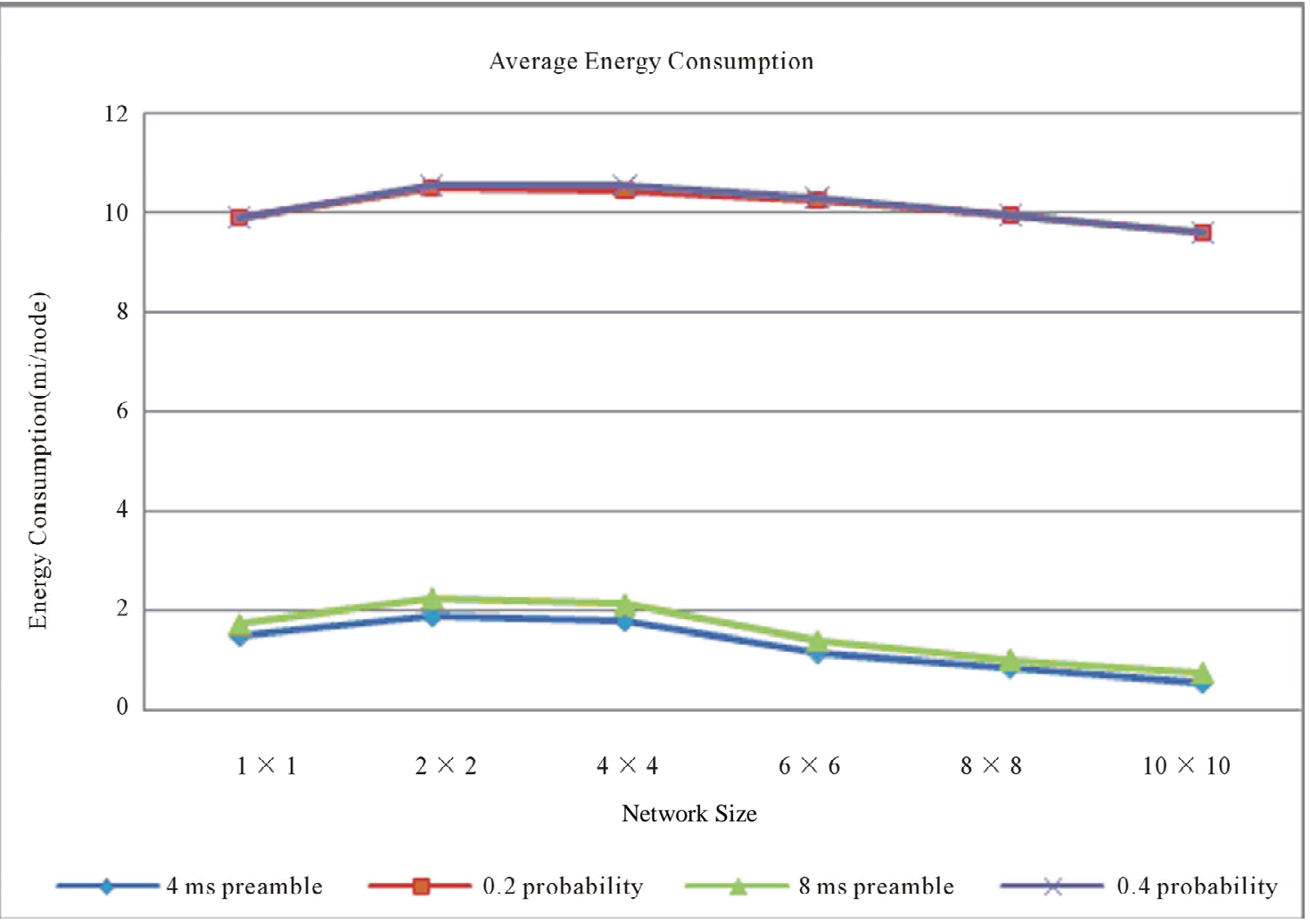

Figure 9. Energy consumption (mj/node) vs network size.

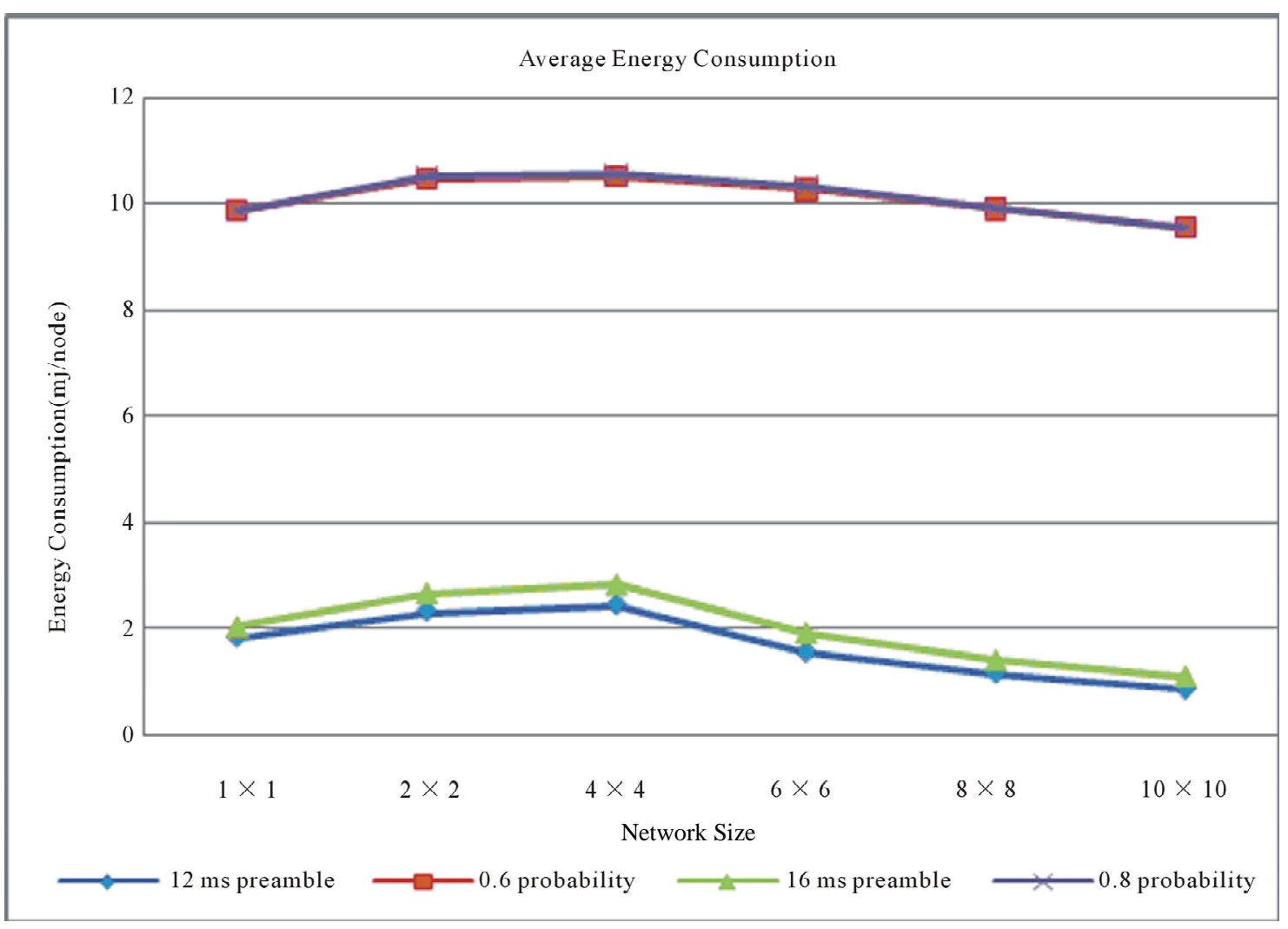

Figure 10. Energy consumption (mj/node) vs network size. 


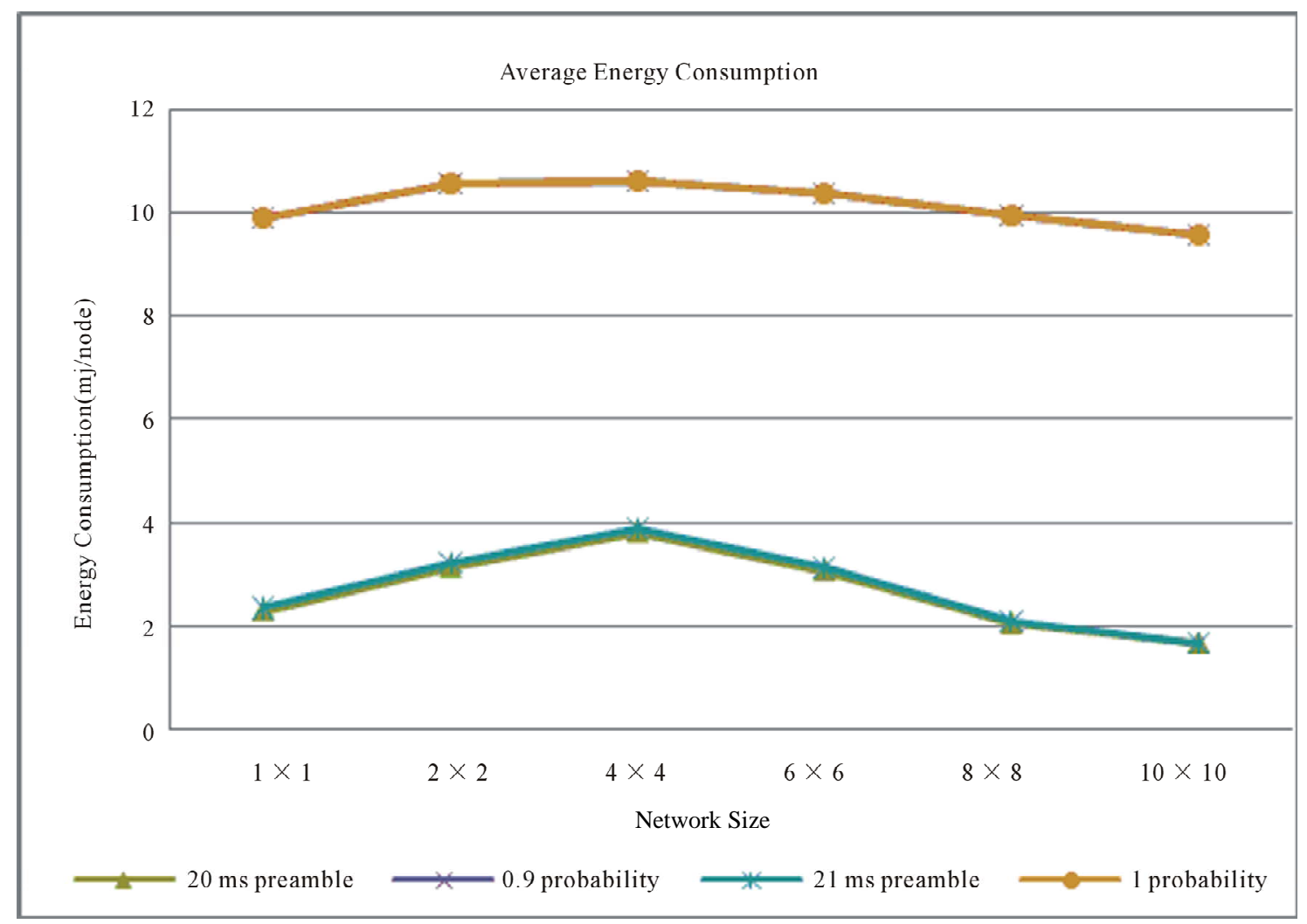

Figure 11. Energy consumption (mj/node) vs network size.

ability in dense area networks and is a better solution in terms of reachability for dense area wireless ad hoc sensors networks. It is observed that our preamble-based scheme achieves significant throughput while consuming less energy and saving significant amount of duplicate packets.

In future, we plan to make an analytical model for our proposed preamble based broadcasting technique in order to compare it with our simulation results. We also plan to make our preamble-based scheme dynamic, so that it can dynamically choose preamble size according to the extra area coverage and neighbor node information.

\section{References}

[1] T. He, S. Krishnamurthy, L. Luo, et al., "VigilNet: An Integrated Sensor Network System for Energy-Efficient Surveillance," ACM Transaction on Sensor Networks, Vol. 2, No. 1, 2006, pp. 1-38. doi: $10.1145 / 1138127.1138128$

[2] L. Selavo, A. Wood, Q. Cao, et al., "LUSTER: Wireless Sensor Network for Environmental Research,” Proceedings of the 5th International Conference on Embedded Networked Sensor Systems, Sydney, 4-9 November 2007, pp. 103-116.

[3] N. Xu, S. Rangwala, K. K. Chintalapudi, et al., "A Wire- less Sensor Network for Structural Monitoring," Proceedings of the 2nd International Conference on Embedded Networked Sensor Systems, Baltimore, 3-5 November 2004, pp. 13-24.

[4] Y.-C. Tseng, S.-Y. Ni, Y.-S. Chen, et al., "The Broadcast Storm Problem in a Mobile Ad Hoc Network," Wireless Networks, Vol. 8, No. 2-3, 2002, pp. 153-167. doi:10.1023/A:1013763825347

[5] B. Williams and T. Camp, "Comparison of Broadcasting Techniques for Mobile Ad Hoc Networks," Proceedings of the 3rd ACM International Symposium on Mobile Ad Hoc Networking \& Amp; Computing, Lausanne, 2002, pp. 194-205.

[6] W. Feng and L. Jiangchuan, "Duty-Cycle-Aware Broadcast in Wireless Sensor Networks,” Proceedings INFOCOM 2009, 19-25 April 2009, pp. 468-476.

[7] S. Guo, Y. Gu, B. Jiang, et al., "Opportunistic Flooding in Low-Duty-Cycle Wireless Sensor Networks with Unreliable Links," Proceedings of the 15th Annual International Conference on Mobile Computing and Networking, Beijing, 20-25 September 2009, pp. 133-144. doi:10.1145/1614320.1614336

[8] Y. Sun, O. Gurewitz, S. Du, et al., “ADB: An Efficient Multihop Broadcast Protocol Based on Asynchronous Duty-Cycling in Wireless Sensor Networks," Proceedings of the 7th ACM Conference on Embedded Networked Sensor Systems, Berkeley, 4-6 November 2009, pp. 43-56. 
[9] S. Yanjun, G. Omer and B. J. David, "RI-MAC: A Receiver-Initiated Asynchronous Duty Cycle MAC Protocol for Dynamic Traffic Loads in Wireless Sensor Networks," Proceedings of the 6th ACM Conference on Embedded Network Sensor Systems, Raleigh, 4-7 November 2008.

[10] A. Kamra, V. Misra and D. Rubenstein, "CountTorrent: Ubiquitous Access to Query Aggregates in Dynamic and Mobile Sensor Networks," Proceedings of the 5th International Conference on Embedded Networked Sensor
Systems, Sydney, 4-9 November 2007, pp. 43-57. doi:10.1145/1322263.1322269

[11] S. Nath, P. B. Gibbons, S. Seshan, et al., "Synopsis Diffusion for Robust Aggregation in Sensor Networks," ACM Transactions on Sensor Networks, Vol. 4, No. 2, pp. 1-40, 2008. doi:10.1145/1340771.1340773

[12] T. Instrument, “2.4 GHz IEEE 802.15.4/ZigBee-ready RF Transceiver,” 2007

http://focus.ti.com/lit/ds/symlink/cc2420.pdf 